\title{
Real-time fluorogenic PCR assays for the detection of entA, the gene encoding staphylococcal enterotoxin $A$
}

\author{
Jennifer R. Horsmon • Cheng J. Cao • \\ Akbar S. Khan · Mark V. Gostomski · \\ James J. Valdes · Kevin P. O'Connell
}

Published online: 10 March 2007

C) Springer Science+Business Media B.V. 2007

\section{Erratum to: Biotechnol Lett (2006) 28: 823-829 DOI 10.1007/s10529-006-9011-0}

There was an error in Table 1 of the original article (the probe and primer sequences did not align to the ent $A$ target sequence). The corrected Table 1 is shown on the following page.

The online version of the original article can be found at http://dx.doi.org/10.1007/s10529-006-9011-0

J. R. Horsmon - C. J. Cao · A. S. Khan .

J. J. Valdes · K. P. O'Connell ( $₫)$

U.S. Army Edgewood Chemical Biological Center, AMSRD-ECB-RT-BM, Aberdeen Proving Ground, 5183, Edgewood, MD 21010, USA

e-mail: kevin.oconnell1@us.army.mil

M. V. Gostomski

SAIC, Aberdeen Proving Ground, P.O. Box 68, Edgewood, MD 21010, USA

C. J. Cao

US Army Center for Health Promotion and Preventive Medicine, Blackhawk Road, E2100/1031, Aberdeen Proving Ground, 5158, Edgewood, MD 21010-5430, USA

\section{A. S. Khan}

Defense Threat Reduction Agency, Fort Belvoir, VA 22060-6201, USA 
Table 1 Primer and probe sequences for real-time fluorogenic PCR assays for the detection of the entA gene of Staphylococcus aureus

\begin{tabular}{lllc}
\hline Assay & Start $^{\mathrm{a}}$ & Probe and Primer sequences $^{\mathrm{b}}$ & Amplicon length (bp) \\
\hline 1 & 297 & Forward: TGCCCTAACGTGGACAACAA & 101 \\
& 397 & Reverse: CTGCTCCCTGCAATTCAGACT & \\
& 320 & Probe: CACTTGTAAATGGTAGCGAGAAAAGCGAAGAAATAA & 99 \\
2 & 714 & Forward: CAATTTATGGCTAGACGGTAAAC & \\
& 812 & Reverse: CTTGCTTGAAGATCCAACTCC & \\
& 741 & Probe: ACAGTACCTTTGGAAACGGTTAAAACGAATAAGAAAA & \\
\hline
\end{tabular}

\footnotetext{
${ }^{a}$ Position of first base in each oligonucleotide sequence relative to the entA sequence in GenBank accession L22566

b All sequences listed in the $5^{\prime}$ to $3^{\prime}$ direction. The $5^{\prime}$ base of each probe was labeled with 6-carboxy-fluorescein (FAM), and the $3^{\prime}$ base of each probe was labeled with 6-carboxytetramethylrhodamine (TAMRA)
} 